\title{
Des humains et des animaux dans L'oeil américain et Le Moineau domestique ${ }^{i i}$
}

\author{
JOSÉE LAPLANTE \\ Université de Sherbrooke
}

\begin{abstract}
Résumé
Cet article propose d'examiner la représentation des rapports humain-animal dans L'œil américain de Pierre Morency et dans Le Moineau domestique de Serge Bouchard en cherchant à dégager les aspects de ces deux textes qui tendent à mettre en évidence le caractère arbitraire de la frontière conceptuelle, érigée par une tradition occidentale, entre humains et animaux.
\end{abstract}

Depuis une trentaine d'années, plusieurs découvertes scientifiques, notamment dans les domaines de l'éthologie, de la paléoanthropologie et des sciences cognitives, tendent à reconfigurer les rapports humain/animal tels qu'ils sont conçus et vécus dans le monde occidental. En effet, la conception dichotomique de la relation entre humain et animal (où l'animal est considéré comme I'Autre de I'homme) ainsi que tout I'appareil d'oppositions qui la sous-tendent (nature/culture; inné/acquis; matériel/spirituel; détermination biologique/libre arbitre) se voient ébranlés par les nouvelles connaissances révélées par divers spécialistes du vivant. Des chercheurs ont montré I'existence d'une conscience et de cultures animales. Ces récents savoirs soulèvent plusieurs questions quant à la frontière entre humain et animal, frontière tracée par une pensée occidentale relativement récente dans I’histoire et qui est, il faut le rappeler, idéologique. Cette frontière relève de constructions idéelles et discursives et elle demeure sujette à des changements selon le contexte. Dans son essai intitulé L'Animal singulier, le philosophe et éthologue Dominique Lestel explique ce qui suit :

Nous nous trouvons donc dans une situation extrêmement intéressante, dans laquelle les caractéristiques par lesquelles nous opposons habituellement l'homme et l'animal se révèlent être des constructions en partie culturelles, certes importantes et respectables, mais qu'il est possible de modifier et de mettre en cause. (2004:69, souligné par l'auteur.)

Lestel défend l'idée de l'existence de cultures chez les animaux et soutient que ceux-ci peuvent être considérés comme de véritables sujets puisque, selon le philosophe, «il n'y a pas de culture sans sujet » (Lestel 2001: 10); cette idée forcément «transforme profondément nos concepts du couple nature/culture, mais aussi ceux du couple homme/animal »(14).

On peut évidemment se demander si les questions soulevées par les sciences du vivant et reconduites par une réflexion philosophique se trouvent relayées dans la production littéraire contemporaine. J'examinerai ici les textes de deux auteurs québécois qui font une place particulière aux figures animales dans leurs écrits. J'aborderai d'abord le premier tome de la trilogie des Histoires naturelles du Nouveau Monde de l'écrivain et naturaliste Pierre Morency. Intitulé L'œil américain, ce texte combine les observations du naturaliste sur la flore et la faune (avec une prédilection pour les animaux, et particulièrement les oiseaux) ainsi que les contemplations et 
les méditations du poète. L'œil américain est largement représentatif de l'ensemble de la trilogie en ce qui a trait à la représentation des rapports humain/animal. En deuxième lieu, c'est l'essai Le Moineau domestique : Histoire de vivre de l'anthropologue et écrivain Serge Bouchard qui retiendra mon attention. Ce recueil est constitué de courts textes portant sur des sujets aussi divers que le téléphone, la mouche, le béton armé, l'épinette noire ou la peine capitale, par exemple. J'examinerai les cinq textes qui portent spécifiquement sur un animal, soit «Éloge du chien », « Le moineau domestique », «La mouche ordinaire », « Les vaches » et « Les loups ». Il est à noter, en outre, que cet essai de Bouchard est le premier d'une série. Ainsi, après Le Moineau domestique, paraîtront L'homme descend de l'ourse (1998) et Les corneilles ne sont pas les épouses des corbeaux (2005). Les titres laissent déjà percevoir la place qu'occupent les figures animales dans l'imaginaire et le discours de l'auteur.

Dans un premier temps, je m'attacherai aux principales caractéristiques de la représentation des rapports humain/animal dans chacun des textes. Dans un deuxième temps, je relèverai les façons dont ces textes problématisent la relation de l'homme à l'Autre-animal; en d'autres mots, je mettrai en lumière les aspects des textes qui tendent à mettre au jour l'arbitraire et les fluctuations possibles de la frontière idéelle érigée entre Nousles humains et Eux-les animaux. J'aurai recours pour cet examen aux idées et concepts fournis par les théories de I'altérité. Si I'on considère, avec Janet Paterson, que « l'Autre n'est pas un concept constant, inaltérable ou invariable, mais une construction idéologique, sociale et discursive sujette à de profondes modifications selon le contexte » (22), et que I'on conçoit, avec Paterson citant cette fois Éric Landowski, qu'«il n'y a pas de frontières naturelles entre le Nous et les Autres, il n'y a que " les démarcations que nous construisons " » (44), alors il apparaît possible d'aborder la question des relations homme/animal par l'intermédiaire des concepts relationnels d'identité et d'altérité. Une tradition occidentale (dans laquelle les écrivains examinés ici s'inscrivent) a construit une identité humaine en grande partie en rapport avec une altérité animale (bien que I'humain soit de fait luimême un animal). Au moment où les critères sur lesquels s'érige ce rapport dichotomique perdent de leur pertinence, la construction - idéelle et discursive - de l'altérité animale devient plus évidente. Alors il peut s'avérer intéressant de se pencher sur les procédés discursifs qui contribuent d'une part à construire l'altérité animale et d'autre part à problématiser ou mettre en cause cette même altérité.

Dans son essai intitulé Figures de l'Autre dans le roman québécois (2004), Janet Paterson entreprend d'examiner les lieux de la construction discursive de l'Autre dans les textes. L'analyse de ces « procédés à l'œuvre dans la mise en discours de l'Autre » (28) guidera le présent travail sur le rapport de I'homme à l'Autre-animal dans les textes de Morency et Bouchard. Ainsi, l'énonciation, l'espace, les descriptions et les stratégies rhétoriques retiendront notre attention dans la mesure où ils servent à construire l'altérité animale ou, par ailleurs, à mettre en évidence cette construction.

\section{L'œil américain : regards sur l'animal sauvage}


Dans l'ensemble de L'œil américain, l'énonciation est assumée par un narrateur (un homme, étroitement lié à la figure de l'auteur) s'exprimant à la première personne. Ce narrateur adopte alternativement, et parfois simultanément, la posture du naturaliste et celle du poète. Les observations et les informations livrées par le naturaliste se mêlent donc à des méditations et envolées lyriques inspirées par la contemplation du monde naturel - au sein duquel s'inscrivent les animaux, parmi toutes les « présences vivantes ». Le rapport humain/animal représenté dans ce premier tome des Histoires naturelles du Nouveau Monde se dessine clairement dès les premières pages, où le narrateur explique sa démarche de naturaliste :

Ce que je veux dire en réalité, c'est que tout être vivant, à quelque règne qu'il appartienne, porte en lui une « extraordinaire jubilation » à laquelle nous sommes invités à puiser. Les voyages sur les crêtes, les traversées du froid, les attentes dans la nuit, les randonnées parmi les moustiques, les stations dans la vase et les piquants, à quoi peuvent-ils bien servir sinon à nous donner d'une plante, d'un animal, d'un oiseau cet éclair qui met le corps en émoi et qui saisit l'esprit d'une ivresse si rare. (OA 21.)

Le « nous » qui apparaît ponctuellement au fil du texte renvoie implicitement, le plus souvent, à une « humanité » ou, plus exactement, à une idée de I'humanité. Celle-ci est évidemment empreinte de subjectivité : subjectivité particulière du narrateur, et subjectivité collective d'une tradition, d'une culture, d'un lieu et d'un temps. Cette humanité impliquée par la première personne du pluriel s'inscrit à distance du monde naturel, et partant, du monde animal. Le passage cité plus haut laisse déjà voir cette distance qu'il s'agit alors pour l'homme de franchir exceptionnellement en faisant l'effort « de sortir de soi, d'aller à la rencontre des choses » $(O A 20)$. $L^{\prime} \ll$ extraordinaire jubilation » présente dans le monde vivant et à laquelle I'homme est invité à puiser va de pair, dans le texte, avec une exubérance vitale de laquelle aussi I'humain semble tenu à distance :

Tout a été découvert, sommes-nous portés à penser dans nos moments de lassitude. Pendant ce tempslà, dehors, une exubérance à chaque seconde se renouvelle, les racines travaillent, les sources montent, les poissons fulgurent dans le torrent, les écorces crient, les feuillages se peuplent de nids, les nids répandent des chants, les gazouillis répondent à des feulements, des plaintes s'enroulent dans les creux du silence, les arbres inventent des musiques, les champs ondulent et crépitent à midi, les fleuves d'odeurs comblent des museaux, [...] (OA 22-23)

La longue énumération de cette envolée lyrique se poursuit pour déployer toute l'exubérance de la vie « naturelle » où aucun élément ne laisse deviner une présence humaine, sinon celle du témoin de cette vitalité foisonnante. Tout au long des Histoires naturelles, un généreux champ sémantique de la vitalité, du foisonnement et de I'exubérance sera développé; I'animal se trouve souvent inscrit dans ce champ sémantique, I'humain ne s'y trouve que marginalement. L'homme est à l'écart de toute cette vie du « dehors » où déborde la vitalité animale; il est néanmoins invité à I'approcher, à y puiser maintes émotions.

En effet, à la jubilation, aux émois et ivresses déjà mentionnés dans le premier extrait cité, s'ajouteront au fil du texte l'enchantement, la fascination, le ravissement, l'émerveillement et des joies diverses qui abondent 
dans le discours du narrateur au sujet de ses contacts avec le monde animal. L'aspect émotif du rapport de I’homme à l'animal est particulièrement important ici. L'émotion est souvent esthétique, et les nombreuses descriptions d'animaux mettent en valeur la beauté et la grâce des couleurs, des formes, des chants, des mouvements. En outre, un lexique du spectacle se développe dans les propos du narrateur si bien qu'il apparaît que, pour une bonne part, le rapport humain/animal peut se lire ainsi : I'animal est spectacle merveilleux, I'homme est spectateur ému. Cette image sous-entend une dichotomie des « espaces » occupés respectivement par I’humain et I'animal.

Il convient à ce moment-ci de remarquer que le monde animal qui fait l'objet du discours du narrateur de L'œil américain est toujours sauvage; l'animal domestique n'y est pas pris en compte. L'animal sauvage est d'autant plus autre qu'il évolue dans un monde étranger au monde humain. Dans le texte intitulé « Un autre monde dans le nôtre », cette étrangeté est énoncée par le narrateur lors d'un commentaire sur les marais où il observe les oiseaux :

ces endroits sont les survivances d'une époque lointaine. Il y a soixante-dix millions d'années, les râles, par exemple, fréquentaient les mêmes milieux qu'aujourd'hui. D'où l'impression renouvelée de faire un mystérieux voyage dans le temps quand on circule à proximité d'un marais. S'il y a un autre monde dans le nôtre, c'est là qu'il se trouve. (OA 39)

Ailleurs encore, le narrateur pose la question : « Les animaux respirent le même air que nous, mais fontils vraiment partie de la même réalité? » (OA 209) Il semble que l'étrangeté de l'animal sauvage, son appartenance à un «autre» monde, donne lieu au « phénomène de projection inhérent à l'altérité » (Paterson 13). Insaisissable dans ses apparitions furtives, farouche, libre, parfois insolite, la figure de l'animal dans L'œil américain fonctionne sensiblement comme celle de l'étranger de passage dont fait état Janet Paterson; elle constitue « un creux à remplir, un réseau de sens à construire, une énigme qui ne peut se résoudre elle-mêmeiii » (64-65) ; elle apparaît comme un vide, un manque, « qui facilite une projection de désirs fantasmatiques» (64). En effet, I'Autre-animal du texte de Morency devient souvent le lieu de projections liées à des déficiences perçues dans le monde humain. Une certaine idéalisation de l'animal (alors pourvu de vertus valorisées positivement) rend celui-ci porteur d'un enseignement auquel I'homme est invité à puiser. Ce phénomène est à l'œuvre, par exemple, dans le passage suivant :

Cette aventure est une de mes meilleures expériences avec les oiseaux de la batture. Une des plus troublantes aussi puisqu'elle m'a permis de frôler la vérité intime de l'oiseau, qui est un être libre, indépendant, farouche, c'est-à-dire insensible aux caprices du premier venu, parce qu'il évolue dans un réel qui coïncide avec le nôtre sans y être tout à fait pareil. Les râles ne nous enseignent pas seulement la patience, ils nous invitent à l'humilité. (OA 35) 
Il faut remarquer en outre que le narrateur soulève explicitement la tendance qu'ont souvent les humains à projeter sur les animaux des qualités qu'ils tiennent pour admirables - et qu'ils jugent leur manquer à euxmêmes. Il évoque

le panache, la couleur, la puissance, la célérité qui nous plaisent tant chez les bêtes sauvages et qui, d'une certaine manière, nous impressionnent, au nom sûrement d'une nostalgie primitive et d'une volonté de puissance que nous projetons inconsciemment sur le règne animal. (OA 177.)

Par ailleurs, on notera que dans l'ensemble du texte, les rapports au monde animal s'énoncent à la première personne (du singulier le plus souvent, du pluriel comme dans le passage cité ci-haut); et c'est presque toujours un sujet humain qui dit I'Autre-animal. Il est rarissime qu'un personnage animal prenne la parole à propos de luimême, des humains ou du monde ${ }^{i v}$. L'animal est objet du discours, et la représentation de la relation de l'humain à I'animal est ordonnée par la subjectivité surplombante du narrateur. Cette subjectivité donne à lire un rapport homme/animal anthropocentré, où I'homme est à distance du monde naturel (et partant du monde animal) qu'il souhaite néanmoins approcher. Ce rapport est fortement marqué par l'émotion (et par l'émotion esthétique en particulier), et l'animal sauvage $s^{\prime} y$ voit attribué maintes qualités qui tendent à l'idéaliser, ceci à travers un phénomène de projection lié à la construction de l'altérité (phénomène soulevé par le narrateur dans son discours sur l'animal sauvage).

Toutefois, le narrateur, depuis son point de vue surplombant, intègre à son propre discours sur les animaux divers autres discours qui deviennent des occasions de présenter différentes représentations du rapport homme/animal. On pourrait dire en effet que c'est sur le plan de la configuration formelle que L'œil américain problématise la question de l'altérité animale. La forme même du texte consiste en un assemblage d'observations scientifiques (de première source ou rapportées), de méditations, d'anecdotes, d'extraits de textes divers (récits des explorateurs du Nouveau Monde, poèmes, carnets de naturalistes, etc.), de contes et légendes appartenant à différents horizons culturels, et d'illustrations. Bien que la subjectivité du narrateur prenne en charge et ordonne I'ensemble hétéroclite de ces discours, la multiplicité des points de vue présentés permet de voir apparaître l'arbitraire et la relativité des catégories d'identité humaine et d'altérité animale telles qu'on les conçoit.

On remarquera ici deux aspects intéressants du texte de Morency. D'abord, cet assemblage hétérogène permet la coexistence de plusieurs modes d'appréhension de la réalité. L'œil américain propose d'approcher le monde animal de diverses façons : I'observation scientifique; la fréquentation régulière de certains animaux dans leur milieu naturel; le folklore animalier avec ses contes, légendes et superstitions; la contemplation et l'expérience lyrique du monde animal; la recherche livresque et l'observation sur le terrain. Déjà, ces multiples manières d'approcher les animaux laissent voir différents processus de construction de l'altérité animale - et parfois certaines de ces approches contribuent à moduler ou mettre en cause cette altérité. 
Ainsi, par exemple, le rapport de I'humain à la chauve-souris apparaît-il bien différent selon le contexte et l'approche qu'il appelle. Dans le cours d'un travail de recherche sur sa physiologie, ses habitudes et ses capacités sensorielles, la chauve-souris est objet de fascination, « une des merveilles les plus accomplies de notre planète » $(O A$ 104). Mais dans le cadre d'une expérience quotidienne de cohabitation non souhaitée, la chauvesouris devient pour l'homme un intrus qu'il faut chasser : «On comprendra que dans les circonstances une seule solution s'imposait: demeurer maître de mon domaine. J'ai dû frapper, les faire tomber, les balayer jusqu'au perron » $(O A$ 108). La merveille de la démarche du naturaliste devient un intrus à abattre dans l'expérience concrète de la territorialité. Cet exemple montre bien, par ailleurs, la différence qui se dessine entre un rapport à l'animal (générique) et un rapport avec un ou des animaux (comme individus). Le rapport de I'humain à la chauvesouris, dont les contours sont tracés par une accumulation d'observations et de connaissances, n'est évidemment pas le même que le rapport d'un homme avec quelques chauves-souris qui se cachent dans sa cabane! Les anecdotes contenues dans le texte de Morency tendent à mettre cet aspect des relations humain/animal en perspective (ce que les observations scientifiques ne mettent pas particulièrement en valeur). De plus, l'intégration dans le fil de ces histoires naturelles de divers contes et légendes mettent en lumière la dimension culturelle de notre rapport au monde animal. La symbolique attachée à chaque animal, les vertus qui leurs sont attribuées, les superstitions dont ils sont l'objet et qui modèlent inévitablement nos perceptions`, sont évoquées grâce aux récits légendaires rapportés par le narrateur.

Ces récits légendaires ponctuant les histoires naturelles permettent par ailleurs de mettre en lumière les constructions culturelles de l'altérité animale d'une deuxième façon: en présentant des visions de la relation humain/animal propres à des horizons géographiques et culturels différents. Dans l'imaginaire de certaines régions d'Europe, la chauve-souris, pour reprendre cet exemple, a été créée par le diable et est présage de mort; en Chine pourtant elle symbolise le bonheur (OA 97). Le conte mettant en scène un hibou (qui, en fait, est un prince sous l'effet d'un sort) relaté dans le texte intitulé « tigre d'en haut » donne à voir la conception d'un rapport homme/animal empreint de magie, un rapport où les frontières entre I'un et l'autre sont poreuses. Et le mythe des Indiens Ojibway au sujet du Grand Lièvre qui fit naître les Hommes (OA 291-292) présente une conception d'une humanité qui partage sa généalogie avec le monde animal.

Ces quelques exemples laissent voir comment la diversité des points de vue qui participe à la configuration formelle du texte de Morency tend à mettre au jour le caractère arbitraire de la frontière entre humain et animal. D'une culture à l'autre, ou d'une approche de la réalité à l'autre à l'intérieur d'un même univers culturel, les significations et les valeurs attribuées aux différences perçues entre humain et animal fluctuent; I'altérité de I'animal apparaît alors elle-même fluctuante. Il semble donc que la représentation des rapports humain/animal construite par le discours subjectif et surplombant du narrateur de L'œil américain soit relativisée 
par les diverses perspectives qui entrent en jeu dans la composition du texte. Une conception principale du rapport de I'humain à l'animal s'impose à travers le texte, mais elle est mise en relation avec d'autres conceptions; le discours du narrateur sur le monde animal s'affiche donc doublement dans sa subjectivité : par l'emploi de la première personne, et par sa mise en rapport avec d'autres discours qui le mettent en perspective.

\section{Le Moineau domestique : composer avec les altérités}

Dès les premières lignes de la quatrième de couverture, l'auteur du Moineau domestique présente son projet littéraire et énonce sa posture particulière face au monde qu'il examine :

Cette revue de l'ordinaire est un exercice continu que je m'impose dans la mesure où je ne comprends rien du monde qui m'entoure. Je n'ai donc d'autres choix que d'interroger sans relâche. Tel un étranger dérouté par la culture dans laquelle il se retrouve, je suis ce naïf obstiné qui examine tout, [...]. (MD, quatrième de couverture.)

C'est donc dire qu'avant même d'avoir ouvert le livre de Serge Bouchard, on sait qu'on se trouve devant un sujet qui se pose à distance du monde qu'il examine, un sujet qui s'énonce lui-même comme « étranger ». Tout au long du recueil, le narrateur réitère cette position particulière et, par ailleurs, il affirme à plusieurs reprises sa préférence pour «le régime de l'à-peu-près», peu valorisé dans «ce monde du tout ou rien » (MD 9). Cette attitude du narrateur importe largement dans l'examen des rapports humain/animal qui nous intéresse ici, car elle indique d'emblée que les relations entre les paradigmes de l'identité et de l'altérité risquent d'être affectées, d'une part par la position d'« étranger » que s'attribue le narrateur et, d'autre part, par ce « régime de l'à-peu-près » qui sera privilégié, au prix d'un abandon au moins partiel des certitudes fondées sur une logique binaire et catégorisante.

Il faut ajouter que les cinq textes portant particulièrement sur des animaux s'inscrivent dans un ensemble de soixante-dix courts textes au fil desquels le narrateur se penche sur divers éléments - concrets ou abstraits - qui font partie du monde qu'il examine. Les animaux sont donc pris en compte ni plus ni moins que tous les autres éléments qui constituent ce monde.

Bien qu'à travers l'ensemble du recueil, et plus particulièrement dans les cinq textes qui retiendront notre attention, une partie de l'énonciation soit assumée par un « nous » qui renvoie plus ou moins explicitement à une idée de l’humanité (ou plus précisément à une humanité moderne occidentale), on ne saurait réduire la représentation du rapport homme/animal à I'opposition entre Nous-les humains et Eux-les animaux. Au contraire, 
chacun des cinq essais portant sur un animal configure un univers où l'énonciation laisse entendre un partage du monde qui n'obéit pas à cette dichotomie homme/animal.

Dans les premiers paragraphes de l'« Éloge du chien » par exemple, le pronom « nous» renvoie au narrateur et à son chien qui marchent et devisent ensemble. L'instance autre de cet univers textuel est alors déplacée vers le personnage du «matérialiste». C'est lui, homme raisonneur, qui se voit affublé du lexique animalisant et réifiant, alors que le chien, dans les paragraphes suivants, se voit attribuer des fonctions humanitaires valorisées positivement : le chien est un « travailleur social », un « guide», un « gardien », un « consolateur ». L'Autre ici est celui qui, excluant les connaissances liées à l'affect et à une expérience de vie partagée avec les chiens, ne perçoit l'animal qu'à la lumière de sa raison univoque.

Le matérialiste myope aurait bien ri de nous voir ainsi deviser, un homme, un chien, par un chaud soir d'été. [...] Oui, le matérialiste aurait bien ri. Mais que sait-il cet animal raisonneur, cette machine triviale, cette taupe frigide qui ne cesse depuis l'âge de raison de se tire-bouchonner un sens unique vers le centre de la terre? Il sait qu'un chien est un chien. Poursuivant dans le domaine des idées, il précisera sans rire que la chose est un animal. Cependant, quiconque a vécu parmi les chiens sait bien que ces êtres-là n'ont d'animal qu'un passé récent sur lequel la plupart préfèrent ne pas revenir. Ceux qui n'en démordent pas et refusent de voir autre chose qu'un chien dans un chien sont ceux-là mêmes qui enseignent que les pierres ne parlent ni ne souffrent. C'est tout dire. (MD 23-24)

L'éloge du chien pour ses qualités humaines et ses actions humanitaires fait de lui un être participant au monde de l'affect et des sentiments; en marge de ce monde, « dans le domaine des idées », I'« animal raisonneur » qu'est le matérialiste fait figure d'Autre méprisé. Au fil de l'éloge, le chien retourne à la troisième personne de la grammaire du texte, néanmoins il conserve les caractéristiques qui l'associent au monde des sentiments humains valorisés dans le discours du narrateur. La différence entre le chien et I'homme tient à la parole, mais cette différence ne devient pas facteur d'altérisation; elle apparaît plutôt comme facteur d'identification, dans une certaine mesure, prenant la forme d'une sorte de fraternité particulière entre I'humain qui parle et le chien qui ne sait pas « s'empêtrer dans le piège des mots » $(M D 25):$ [Le] chien est le premier et le plus fidèle compagnon esseulé. Il accompagne le vieillard inquiet le long de ses derniers sentiers. Mieux encore, tout près de son lit de mort, la présence d'un chien épargne bien des paroles inutiles » (MD 24). Dans un monde humain affligé de souffrances (un champ sémantique de la faiblesse et de la douleur se déploie pour évoquer la condition humaine), le chien offre généreusement sa compagnie et son affection.

On remarquera évidemment que l'identification entre l'humain et le chien passe ici par une valorisation de l'affect, peut-être au prix d'une négation de la raison. Toutefois, en gardant à l'esprit l'attitude affichée par le narrateur dès le début du recueil à l'endroit du «monde du tout ou rien», on peut supposer que cette valorisation de l'affect irait plutôt de pair avec une dévalorisation d'une raison unique, univoque. En d'autres mots, le discours du narrateur en appelle à une approche ouverte, multiple, variée du monde. On notera que si le matérialiste raisonneur à sens unique est dévalorisé, la figure du penseur qui tourne autour d'un sujet est, elle, valorisée. On 
remarquera en outre que le narrateur attribue au chien des talents qui ne sont pas uniquement de l'ordre de l'affect, mais aussi de la réflexion : «Les chiens sont des philosophes, des praticiens de l'affection, des cœurs à quatre pattes » (MD 25, je souligne). Le chien tel qu'il apparaît dans les propos du narrateur est un compagnon avec qui I'homme partage sa vie; de là, il est possible de l'identifier au monde humain. L'homme et le chien (à l'instar de tous les animaux par ailleurs) partagent ensemble une communication de l'ordre de l'affect, et cette dimension affective et émotionnelle n'est jamais à exclure ${ }^{\text {vi }}$; il semble que ce soit cette dimension que le narrateur ait choisi de privilégier pour construire un rapport d'identification entre homme et animal.

Une relation d'identification entre humain et animal opère aussi, mais d'une façon différente, dans le texte qui donne son titre au recueil. «Le moineau domestique» repose sur une analogie entre I'humain et le moineau, analogie qui s'arrime à la médiocrité attribuée aux deux espèces : « Nous sommes tous des moineaux domestiques; petits, sales, résistants, bourrés de frites et d'illusions, nous survivons aux hivers pour mieux mourir au printemps, nous rusons le chat et le destin pour un tout qui, à la fin, n'est rien » (MD 47) Alors la triste description de la vie et des gestes des moineaux devient celle de la vie des humains depuis I'analogie annoncée. Un lexique de la maladie, de la saleté et de la contagion fait du moineau - et donc de I'homme - une « domestique vermine » dont le destin annoncé est celui d'une extermination, qui de surcroît émanera d'elle-même. On notera que la rhétorique de ce texte s'appuie entièrement sur une idée d'animalité entendue dans sa plus négative acception. L'animalité du moineau domestique - et de I'homme - renvoie ici à la bassesse, à la médiocrité, à la vulgarité, à la saleté, ultimement à la maladie contagieuse et à la mort. L'opposition entre les idées extrêmes d'humanité et d'animalité qui structure toute une tradition philosophique occidentale (Picq 2007, 80-83) sert ici à mettre en discours une critique sévère de I'humanité, en I'identifiant au dernier degré de l'animalité telle qu'elle I'a elle-même conçue, I'ultime Autre : la vermine à exterminer.

Les textes sur le chien et sur le moineau domestique montrent à eux seuls que les stratégies d'énonciation et les procédés rhétoriques contribuent, dans l'essai de Serge Bouchard, à mettre en discours des rapports complexes et multiples entre humains et animaux, rapports qui débordent la dichotomie Nous-les humains/Eux-les animaux. On remarquera par ailleurs que les processus d'identification mis en œuvre dans les deux textes examinés peuvent tout autant servir une valorisation qu'une vision négative de l'animal-humain construit dans le discours du narrateur.

Si dans les Histoires naturelles de Morency c'est I'animal sauvage qui occupe le discours, dans les essais de Bouchard c'est l'animal familier ou domestique, l'animal commun, qui occupe la plus grande part des réflexions du narrateur. Quatre des cinq animaux qui font l'objet d'un texte s'inscrivent dans I'univers familier des humains. L'espace partagé par les hommes et les animaux y est celui de l'ordinaire : la maison et ses alentours; la ville, ses rues, ses trottoirs et stationnements; la campagne agricole et la ferme. Ces lieux de I'ordinaire humain sont 
peuplés d'animaux, mais la coexistence au quotidien ne va pas nécessairement de pair avec la (re)-connaissance de I'animal. Le narrateur signale la méconnaissance et le mépris que I’homme voue à certains animaux familiers, dans les textes intitulés « La mouche ordinaire » et « Les vaches ». Ces deux textes donnent à lire une méconnaissance et un mépris de l'animal familier qui se présente en quelque sorte comme l'envers de l'idéalisation qu'inspire souvent chez I'humain l'animal sauvage. On l'a vu dans L'œil américain, l'altérité de l'animal sauvage qui semble appartenir à un monde étranger devient l'occasion d'une certaine idéalisation et de projections fantasmatiques. Au contraire, l'animal domestique évolue dans un monde connu de I'humain, un monde rempli d'objets, de contraintes, d'espaces qu'ils partagent.

Dans «La mouche ordinaire » et « Les vaches », le narrateur soulève les diverses manifestations du mépris humain pour ces bêtes communes, puis il tente de proposer une perspective différente afin de poser un regard plus généreux sur ces animaux. Ainsi, il se rappelle les tortures infligées aux mouches ordinaires lors de jeux enfantins cruels. Il évoque les caractéristiques et les comportements de ces insectes qui les rendent repoussantes aux yeux de plusieurs. Puis il s'efforce de montrer les mérites de cette bête mal aimée — quitte à en imaginer - pour finalement tenter de présenter la mouche sous un jour qui saurait inspirer le respect : « Autant d'énergie, de complexité, dans un petit point noir qui explore l'espace avec pareille agilité, cela commande le respect. Chacune est un chef-d'œuvre et on les compte par millions de milliers » (MD 85).

Une réflexion semblable préside au discours sur les vaches. Présentée dans ses habitudes de domesticité et de promiscuité un peu malpropre, prisonnière de l'industrie humaine, ne cherchant manifestement pas à s'évader des enclos qu'on lui assigne, la vache a « un je-ne-sais-quoi de résigné qui n'est pas pour l'avantager sous le rapport de sa personnalité »(MD 111). Et pour cause, la vache ici décrite évolue aux antipodes du monde de l'animal sauvage et idéalisé, celui fier, noble et souverain des fantasmes humains de grande liberté. Encore une fois, le narrateur s'efforce ensuite de présenter l'animal familier un peu méprisé sous un jour différent :

Les vaches qui, depuis maintenant des millénaires, observent les éleveurs et les regardent faire, ont sûrement sur le sujet une petite idée. Si elles ont l'air nerveuses et bêtes, c'est peut-être qu'elles redoutent le jour où elles auraient à l'exprimer. Il y $a$, dans l'œil de la vache, un soupçon d'ironie et bien qu'elle soit manifestement nulle en mathématique, en histoire et en géographie, il apparaît, pour qui sait y voir, qu'elle est passée maîtresse en matière de culture et en philosophie. (MD 112-113)

Ici, comme c'était le cas avec le chien, la conscience et la pensée « philosophique » de l'animal sont convoquées par le narrateur pour établir des correspondances entre homme et animal. Dans l'ensemble des cinq textes examinés ici, d'ailleurs, il semble que les rapports humain/animal instaurés par le narrateur portent la marque d'un processus d'identification qui passe, selon le cas, par une conscience partagée, un affect partagé, des caractéristiques ou des espaces partagés. 
Un seul texte de ce recueil porte sur un animal sauvage, et c'est celui qui met en œuvre le plus visiblement et ouvertement une problématisation de l'altérité animale. « Le loup » explore la construction discursive de la fascination doublée de crainte qu'inspire cet animal sauvage. Il faut remarquer d'emblée que le loup se caractérise dans le texte par sa non-présence dans le monde humain; les loups « font des cercles autour des cirques humains, n’y pénétrant jamais, s'en éloignant toujours » (MD 177). Le loup constitue donc une figure étrangère et furtive qui devient un lieu de projection. Ce que Janet Paterson explique à propos de la figure de l'étranger de passage qui apparaît comme « un creux à remplir, un réseau de sens à construire » (64-65), Serge Bouchard l'exprime en d'autres termes à propos du loup qui « protège son image en ne la montrant pas. Le loup étant un adepte de la vie courante, il sait depuis un bail que l'impression la plus forte est l'impression la plus fuyante. Il faut qu'on l'imagine celui qu'on ne voit pas » (MD 176, je souligne).

Alors le narrateur montre comment I'on glisse du loup au mot loup puis aux histoires de loups, investissant d'un sens terrible l'animal sauvage à peine perçu : « c'est cela le loup, un simple mot qui mord, un petit mot de rien dont le sens nous dévore »(MD 176). Les loups les plus terrifiants sont ceux qui «courent et trottinent sur les sentiers de notre imaginaire » (176), ce qui fait dire au narrateur: « S'il fallait en croire et nos récits et nos histoires, nous n'aurions pas fini de parler de nos cicatrices, de nos morceaux perdus, de ces vilaines morsures laissées par l'incisive de la mémoire » (176).

\section{Conclusion}

Les textes de Pierre Morency et de Serge Bouchard instaurent, chacun à sa manière, des rapports entre humain et animal qui peuvent être appréhendés à l'aide des concepts relationnels d'identité et d'altérité. Ces écrits mettent en œuvre des procédés formels ou discursifs qui tendent à mettre en lumière l'arbitraire qui entre en jeu dans les conceptions de ce qui lie ou sépare les humains des animaux non humains. Le rapport d'un sujet humain au monde animal dans la narration de L'œil américain s'inscrit dans une conception dichotomique où l'humain apparaît à distance du monde naturel (et partant du monde animal) qu'il s'agit alors d'approcher afin d'y puiser émerveillement et émotion. Ce rapport est fortement marqué par l'émotion esthétique et le monde animal apparaît au fil du texte comme un spectacle le plus souvent enchanteur. L'animal sauvage occupe seul les observations et méditations du narrateur; il est presque exclusivement l'objet du discours d'une subjectivité humaine. Par ailleurs, cette conception principale qui préside à la représentation de l'animal et des rapports humain/animal est modulée dans le texte par la configuration formelle de ces histoires naturelles qui sont composées d'un assemblage hétéroclite de divers discours. La forme du texte donne ainsi à voir la relativité des conceptions des relations humain/animal. Par ailleurs, dans Le Moineau domestique, le rapport humain/animal apparaît d'emblée comme le 
lieu d'une interrogation sur les mécanismes de construction de l'altérité. Les divers procédés discursifs et rhétoriques utilisés dans les cinq textes portant spécifiquement sur un animal donnent à lire des rapports entre hommes et animaux qui excèdent la dichotomie classique entre Nous-les humains et Eux-les animaux. Des processus d'identification à l'œuvre dans le discours du narrateur tendent à rapprocher le monde animal et le monde humain, en mettant en valeur des aspects partagés par l'un et l'autre. Des textes portant sur les animaux familiers abordent la question du mépris qui parfois imprègne les rapports de l'homme avec les animaux domestiques (évoluant dans des espaces et contextes qui lui sont familiers). Le seul texte portant sur un animal sauvage met au jour les constructions discursives qui participent à la fabrication de l'Autre terrifiant.

On peut constater finalement que les démarches littéraires distinctes de Pierre Morency et de Serge Bouchard participent, chacune à sa manière, à questionner les relations et les frontières entre humains et animaux qui se construisent dans l'imaginaire, dans les discours, dans la pensée et les expériences de la réalité. Un examen plus complet qui tiendrait compte de l'ensemble de la trilogie des Histoires naturelles du Nouveau Monde de Morency et des autres essais de Bouchard parus depuis Le Moineau domestique saurait probablement révéler des résultats plus complets que ceux obtenus dans le cadre de la présente analyse. Peut-être, notamment, que des changements dans la représentation des rapports humain/animal apparaîtraient au fil d'un examen diachronique des textes (compte tenu de la diffusion, au cours des années 1990 et 2000, des savoirs sur les mondes mentaux et sociaux des animaux). Dans le cas de Serge Bouchard, il serait pertinent en outre de se pencher, dans le cadre de la problématique abordée ici, sur ses Confessions animales (2006). Ce texte qui donne la parole tour à tour aux animaux sauvages de la faune nord-américaine convertit un genre littéraire propre à l'expression de subjectivités humaines pour donner à lire des subjectivités animales. Les effets de ce procédé littéraire sur la représentation des rapports humain/animal seraient intéressants à examiner, puisque c'est la parole de l'Autre qui ultimement dans les textes met en cause l'altérité qu'on lui assigne.

http://www.brocku.ca/cfra/voixplurielles05-02/index.html 


\section{Références}

BOUCHARD, Serge (1998), L'homme descend de l'ourse, Montréal, Boréal.

BOUCHARD, Serge ([1991] 2000), Le Moineau domestique : Histoire de vivre, Montréal, Boréal.

BOUCHARD, Serge (2005), Les corneilles ne sont pas les épouses des corbeaux, Montréal, Boréal, coll. « Papiers collés ».

FONTENAY, Élisabeth de (1998), Le silence des bêtes. La philosophie à l'épreuve de l'animalité, Paris, Fayard.

LANDOWSKI, Éric (1997), Présences de l'autre, PUF, « Formes sémiotiques ».

LESTEL, Dominique (2001), Les Origines animales de la culture, Paris, Flammarion.

LESTEL, Dominique (2004), L'Animal singulier, Paris, Seuil.

MORENCY, Pierre (1989), L'œil américain, Histoires naturelles du Nouveau Monde, illustrations de Pierre Lussier, préface de Jean-Jacques Brochier, Boréal/Seuil.

MORENCY, Pierre (1992), Lumière des oiseaux, Histoires naturelles du Nouveau Monde, illustrations de Pierre Lussier, préface d'Yves Berger, Boréal/Seuil.

MORENCY, Pierre (1996), La Vie entière, Histoires naturelles du Nouveau Monde, illustrations de Pierre Lussier, Boréal/Seuil.

PATERSON, Janet (2004), Figures de l'Autre dans le roman québécois, Nota bene, coll. « Littératures(s) 》.

PICQ, Pascal, Boris Cyrulnik, Jean-Pierre Digard et Karine-Lou Matignon (2000), La plus belle histoire des animaux, Paris, Seuil, coll. «Points»

PICQ, Pascal (2007), Nouvelle histoire de l'Homme, Perrin, coll. «tempus». 

i Pierre Morency, L'œil américain, Histoires naturelles du Nouveau Monde, préface de Jean-Jacques Brochier,
illustrations de Pierre Lussier, Montréal et Paris, Boréal et Seuil, 1989. Les références à cet ouvrage seront
indiquées par le sigle $O A$, suivi de la page, et placées entre parenthèses dans le corps du texte.

i Serge Bouchard, Le Moineau domestique : Histoire de vivre, Montréal, Boréal, 2000 [1991]. Les références à cet ouvrage seront indiquées par le sigle $M D$, suivi de la page, et placées entre parenthèses dans le corps du texte.

iii Il est à noter que les champs sémantiques de l'énigme et du mystère sont présents dans le discours du narrateur au sujet du monde animal.

iv Quelques rares et brèves occurrences font exception, notamment dans le cadre d'un récit légendaire rapporté par le narrateur aux pages 244 à 246, et dans un passage sur le grillon à la page 313. Il est à noter en outre que les deuxième et troisième tomes de la trilogie présentent un peu plus de procédés discursifs qui donnent la parole aux animaux que le premier tome.

` Même les observateurs scientifiques n'échappent pas à la prégnance de ces préjugés culturels liés à toute une imagerie populaire peuplée d'animaux. Dominique Lestel explique à ce sujet : « La réputation d'un animal peut constituer une difficulté supplémentaire à la juste compréhension de ses comportements et de ses états mentaux. Même s'il se veut indépendant des préjugés de sa culture, l'éthologue est influencé par le regard que les autres posent sur l'objet de ses recherches, et s'en démarquer constitue pour lui un objectif prioritaire » (2004: 45).

vi «Pour le psychiatre phénoménologue Erwin Strauss, l'affect est ce qui rapproche vraiment I'homme et l'animal. Nous sentons de façon mutuellement traduisible. Ce que I'homme comprend, c'est ce que l'animal ressent, parce que I'homme le ressent lui aussi de façon proche » (Lestel $2004: 79$ ). 